\title{
Multilayer Haptic Feedback for Pen-Based Tablet Interaction
}

\author{
Ernst Kruijff \\ Institute of Visual Computing \\ Bonn-Rhein-Sieg University of \\ Applied Sciences \\ Sankt Augustin, Germany \\ ernst.kruijff@h-brs.de \\ Jens Maiero \\ Institute of Visual Computing \\ Bonn-Rhein-Sieg University of \\ Applied Sciences \\ Sankt Augustin, Germany \\ jens.maiero@h-brs.de
}

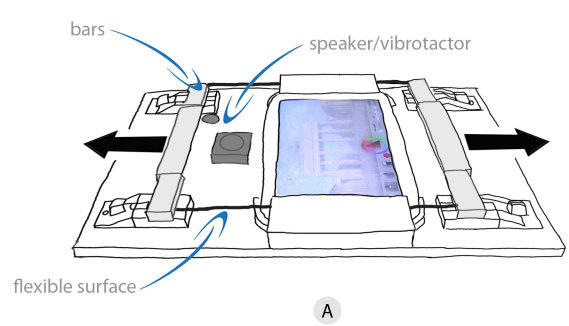

Saugata Biswas

Institute of Visual Computing

Bonn-Rhein-Sieg University of

Applied Sciences

Sankt Augustin, Germany

saugata.biswas@h-brs.de

\author{
George Ghinea \\ College of Engineering, Design and \\ Physical Sciences \\ Brunel University London \\ London, United Kingdom \\ george.ghinea@brunel.ac.uk
}

\author{
Christina Trepkowski \\ Institute of Visual Computing \\ Bonn-Rhein-Sieg University of \\ Applied Sciences \\ Sankt Augustin, Germany \\ christina.trepkowski@h-brs.de
}

\section{Wolfgang Stuerzlinger \\ School of Interactive Arts + \\ Technology \\ Simon Fraser University \\ Vancouver, BC, Canada w.s@sfu.ca}

Figure 1. Our new FleXurface multilayer system consists of a stretchable flexible surface (a flexible haptic layer) suspended above a tablet display (a rigid screen layer). The flexible layer enables exploration of, or guidance within, haptic features of the interactive content displayed on the tablet. (A) The bars (light grey) stretch the flexible surface to change its haptic properties, which can be further augmented through vibration (dark grey: speaker, vibrotactor). (B) The user can press down on the flexible surface to explore the haptic properties. (C) This enables the simulation of, e.g., different brushes (firmness) and paper surfaces (texture, through vibration).

\section{ABSTRACT}

We present a novel, haptic multilayer interaction approach that enables state transitions between spatially abovescreen and 2D on-screen feedback layers. This approach supports the exploration of haptic features that are hard to simulate using rigid $2 \mathrm{D}$ screens. We accomplish this by adding a haptic layer above the screen that can be actuated and interacted with (pressed on) while the user interacts with on-screen content using pen input. The haptic layer provides variable firmness and contour feedback, while its membrane functionality affords additional tactile cues like

Permission to make digital or hard copies of all or part of this work for personal or classroom use is granted without fee provided that copies are not made or distributed for profit or commercial advantage and that copies bear this notice and the full citation on the first page. Copyrights for components of this work owned by others than ACM must be honored. Abstracting with credit is permitted. To copy otherwise, or republish, to post on servers or to redistribute to lists, requires prior specific permission and/or a fee. Request permissions

from Permissions@acm.org.

CHI 2019, May 4-9, 2019, Glasgow, Scotland UK (C) 2019 Association for Computing Machinery. ACM ISBN 978-1-4503-5970-2/19/05 ...\$15.00

https://doi.org/10.1145/3290605.3300373 texture feedback. Through two user studies, we look at how users can use the layer in haptic exploration tasks, showing that users can discriminate well between different firmness levels, and can perceive object contour characteristics. Demonstrated also through an art application, the results show the potential of multilayer feedback to extend onscreen content with additional widget, tool and surface properties, as well as for user guidance.

\section{CS CONCEPTS}

H.5.2 User Interfaces: auditory feedback, Haptic I/O, interaction styles.

\section{KEYWORDS}

Multilayer interaction; pen interaction; haptic feedback.

\section{ACM Reference Format:}

Ernst Kruijff, Saugata Biswas, Christina Trepkowski, Jens Maiero, George Ghinea, Wolfgang Stuerzlinger. 2019. Multi-Layer Haptic Feedback for Pen-Based Tablet Interaction. In 2019 CHI Conference on Human Factors in Computing Systems Proceedings (CHI 2019), May 4-9, 2019, Glasgow, Scotland, UK. ACM, New York, NY, USA. 14 pages. https://doi.org/10.1145/3290605.3300373 

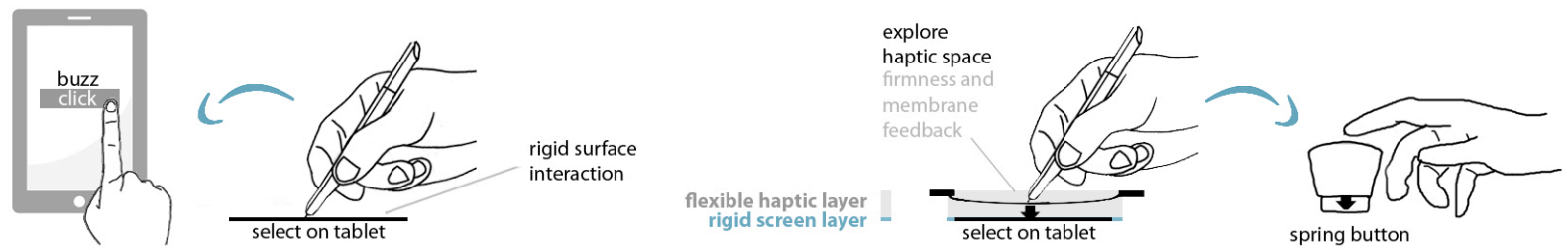

Figure 2. Button example for multilayer interaction: (Left) traditional mobile interaction, where clicking a button on a screen only affords constrained feedback (in comparison to real interaction) as the button cannot be depressed - only a click sound or vibration can be provided; variable compliant haptic exploration allows perception of button properties in $3 \mathrm{D}$ space ("spatial haptic layer"), before the selection (clicking) of the button on the rigid screen (layer), which constraints the downward motion once the button has been selected, similar to a spring button (Right). In the latter example, variable compliant and constrained feedback are used to transition between flexible and rigid states.

\section{INTRODUCTION AND MOTIVATION}

In everyday life, we regularly explore different kinds of haptic properties. For example, we interact with a wide variety of buttons, nobs and tools, which have each a distinct shape and feel. Not only does this "look-and-feel" provide cues on how to operate a specific control or tool, it may also help us to use tools eyes-free [8]. Haptic feature exploration typically includes the probing of kinesthetic and tactile cues such as texture, firmness, temperature, weight, enclosure and contours [46]. During real-world interaction, we might explore haptic features constrained by a surface. Often these features are related to material properties, e.g., texture [47]. However, we might also explore features in a more spatial manner. For example, we might pass our finger across the contour of an object or experience the springiness of an elastic tool, such as a brush (Fig. 1 and 2). Some material probing tasks also exhibit limited spatial characteristics, e.g., we press down on a surface to experience its firmness through displacement of the material and/or the finger that explores the material [24].

Haptic features have been shown to improve user performance [40]. Tactile cues - which can substitute kinesthetic ones - can enhance performance [6], improving expressiveness [4,75], perceptual accuracy and nonambiguity [50]. However, not all haptic properties can be transferred during sensory substitution [35]. The rigid nature of a tablet screen further limits exploration of spatial and elastic properties. The question thus is, how one can support haptic feature perception during tablet interaction.

Multilayer haptic interaction [74]. In this paper we present a novel multilayer interaction approach for penbased tablet interaction. A haptic layer supports the exploration of haptic features and direct manipulation of (visual) screen content. We use an actuated haptic layer suspended above a tablet screen (spatial "flexible haptic layer", above-screen), which the user interacts with via a non-tethered pen (Fig. 1, 4). Visual content on the tablet screen (constrained "rigid screen layer", on-screen) is aligned with the haptic layer.

We control the firmness of the flexible haptic layer by stretching it, affording variable compliant haptic feedback $[38,69]$ without the need for sensory substitution (Fig. 1, 4). We also use the membrane properties of the haptic layer to relay vibration, typically in cohesion with audio cues. Previous work has shown high relevance of vibro- and audio-tactile cues for improving performance $[6,29,51,52,59,62,79]$. The motivation for combining audio and tactile cues is that perception research has shown that sound can enhance touch perception $[23,66]$, e.g., during mobile [31] and haptic interaction [19], and specifically also for texture perception [41], which is highly relevant for the art application presented in the second half of this paper.

Due to the gap between the flexible haptic layer and the tablet screen, the tracked pen can be pressed down towards the screen. As such, we support non-tethered spatial exploration of the trackable space above the 2D display, while still enabling haptic feedback on the pen. Users can easily transition between both layers, as with sufficient pen pressure the haptic layer under low tension can touch the rigid screen. The transition between flexible and rigid states is an important feature of our approach: such transitions are often used by engineers and designers during device, tool and system usage, see a discussion about state changes and shape-changing interfaces in [20].

Our new multilayer system supports the exploration of material and spatial haptic features in both flexible and rigid "space". With our system, we can simulate firmness and shape contours using the spatial and flexible characteristics of the haptic layer. We can also simulate texture using the membrane's properties, which can generate spatial feedback, as the surface moves slightly with vibrations (Fig. 8C/D). However, our approach can also simulate other material properties, such as the continuous feedback associated with soft tools (Fig. 1). Finally, the rigid layer (tablet screen) displays the visual output and is used for direct manipulation, while it can also constrain pen 
motion to a $2 \mathrm{D}$ plane. We thus can mimic a real-world event, such as physically pressing a soft or "spring-force" button before actually activating that button, i.e., state transitions between flexible and rigid states (Fig. 2). Below, we will show that the haptic cues afforded by our system have high relevance for interaction and illustrate the different dependencies between the feedback layers. We do so through two user studies and an exemplar art application.

\section{RELATED WORK}

Above-display and mid-air feedback. The interaction space above displays has been explored in various "hover space" approaches $[2,12,54,64]$. Haptic feedback to hands in mid-air has also been studied, using ultrasound [10], air [43,70] or magnetic fields [58,81]. Our approach opens a new kind of multilayer interaction space, extending previous above-the-table multilayer approaches [74] by adding the exploration of haptic properties in the spatial flexible haptic layer. In contrast to both non-contact-based and mechanically linked systems, our system extends hover space approaches through contact-based variable compliant haptic feedback. We principally divide above-the-display space into digital (virtual) and physical space. Hover space generally explores digital objects in a digital space, whereas our work looks at digital objects in a physical space.

Deformable surfaces. Researchers have studied general interaction metaphors with deformable materials [32,49,68], the use of electromagnets [33] or bendable materials, including paper [22] or silicon(-like) materials [28], and simulating variable compliance on rigid screens [38]. Flexpad used a tracked flexible and highly deformable surface [71]. However, results were strongly tied to specific tasks and not easily generalizable. Deformable and projectable variable compliant surfaces have been explored [60], but were not always well-controllable [61]. Other kinds of materials, including sand and fluids, have also been studied, but are also difficult to control [20]. In contrast, jamming interfaces [20] and Tablehop [67] provide flexible and shape-changing user interfaces with controllable material stiffness (firmness), which are also suitable for mobile devices [20]. This approach illustrates some of the potential of haptic feedback through controllable stiffness. Similar feedback has also been explored in mechanical actuation for handheld squeezing [25], bendable screens [72], and mouse-like interfaces [57], as well as medical skin simulation $[3,42]$. We progress beyond the state of the art through advances in actuation of a flexible surface and combining the tension feedback with rigid and audio-tactile cues to enhance $2 \mathrm{D}$ interaction.

Audio-tactile. Our work is also related to various studies that looked at tactile technologies [27]. Currently, tactile

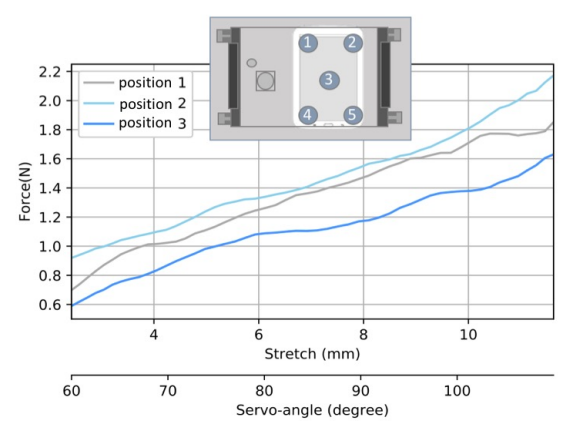

Figure 3. Stress-strain curves at 3 measured positions $(1,2,3)$. Due to symmetry, positions 1 and 4 , and 2 and 5 produce approximately the same results.

cues are almost exclusively based on vibration [29]. They often are employed to reduce visual or auditory attention, enabling eyes-off interaction, as they operate in a separate perceptual channel. Here, visual information is often translated into tactile cues, a process known as sensory substitution [35]. Vibrotactile cues have been shown to improve task performance [62], for example for virtual keyboards [6] or button "clicks" [21]. Some studies also demonstrated task performance improvements under cognitive load [51]. Vibrotactile cues have also been used to simulate various surfaces $[29,65,73]$, friction characteristics [52,59], and rims associated with surface textures [39]. Furthermore, an increase in vibration feedback has been shown to increase perceived softness [79]. Finally, adding audio cues to tactile or haptic feedback has been studied, by looking at which modality works best for widgets [30] or shapes [9], or to guide users, e.g., in 3D environments $[55,56]$. Other studies looked into the interplay of audio and haptic cues for touch screen interaction [77]. Our work advances the current state of the art by investigating how tactile and audio-tactile cues can be used in combination with a flexible surface.

Pen interaction. Haptic and tactile cues have been explored in pen interaction on screens $[13,48,80]$, including those that provide non-controllable, sideways elastic feedback [26]. Pen-based tactile cues improve user performance (affected by a learning-curve [51]), especially for smaller targets [63]. General interaction with touchscreens $[11,45,48]$, or artistic and writing applications $[4,13,18]$ and education [53] have been explored. These systems often provide feedback similar to haptic mice [1] or pens for spatial interaction $[17,37,44]$. Finally, there are some handheld (joystick-scale) devices that provide texture [5] and variable compliant haptic [78] feedback, but these are not useable for tablet interaction. Our work differs substantially from the various haptic pens that have been proposed previously. Such pens often require physical 
contact with a rigid screen to explore physical aspects, or only provide basic force feedback while being operated in mid-air. In essence, it is very difficult to afford our form of combined haptic feedback into a pen in a small form-factor: most pens only support a subset of our functionality, and are often tethered.

\section{SYSTEM}

The main premise behind our system, called FleXurface, is the combination of a transparent, variable-tension flexible material with a tablet computer. By stretching the flexible material, we can achieve different levels of tension. The tablet screen is used for information display, interaction purposes, and for rigid feedback (Fig. 1,4). The physical system consists of high-density wood and 3D printed parts that hold the various actuators. The system is driven by a Unity3D client-server system. The server, the tablet, communicates with the client, a Windows 10 laptop computer, over Wi-Fi. The various actuators, i.e., the servos (servomotors) and the vibrotactor, are controlled by the laptop through an Arduino Mega.

Display. For the main display we use a Samsung Galaxy Tab 8 tablet, which offers an 8 " touch screen. It natively supports interaction through a pen, the S-pen (which has 93 ms latency, see our discussion section), as well as through direct contact using fingers. The pen's 2D location relative to the screen can be tracked if the pen-tip stays within approximately $12 \mathrm{~mm}$ of the display. As the S-pen is pressure sensitive, we measure the relative pressure placed on the tip of the pen for interaction purposes. Pressure sensing is reliable at all tension levels: measurements were made at $6 \mathrm{~mm}$ penetration depth under all tension levels (Fig. 3). For example, we are able to trigger a button feedback reliable at the same penetration depth under all tension conditions, which is important for replicability.

Flexible haptic layer. We use a fully-transparent $0.8 \mathrm{~mm}$ silicon sheet as the actuated flexible surface (Silex Superclear 40 shore). It is glass-clear so that the content presented on the tablet screen is fully visible. The flexible material is mounted between two bars, which stretch the material over the tablet display. Orthogonal to the flexible material, a bendable ultra-thin plastic layer is mounted so that it lies directly on top of the flexible material. This is the layer that the pen presses directly on. This layer is necessary to protect the flexible material and to reduce friction with the pen: the flexible material itself presents too much friction, which makes sliding with the pen difficult. The plastic layer is fixed on the sides using highly flexible neoprene, which allows this layer to flex together with the flexible material. The material is wider than the display area to avoid the appearance of wrinkles. During our experiments, no such wrinkles appeared over the display.

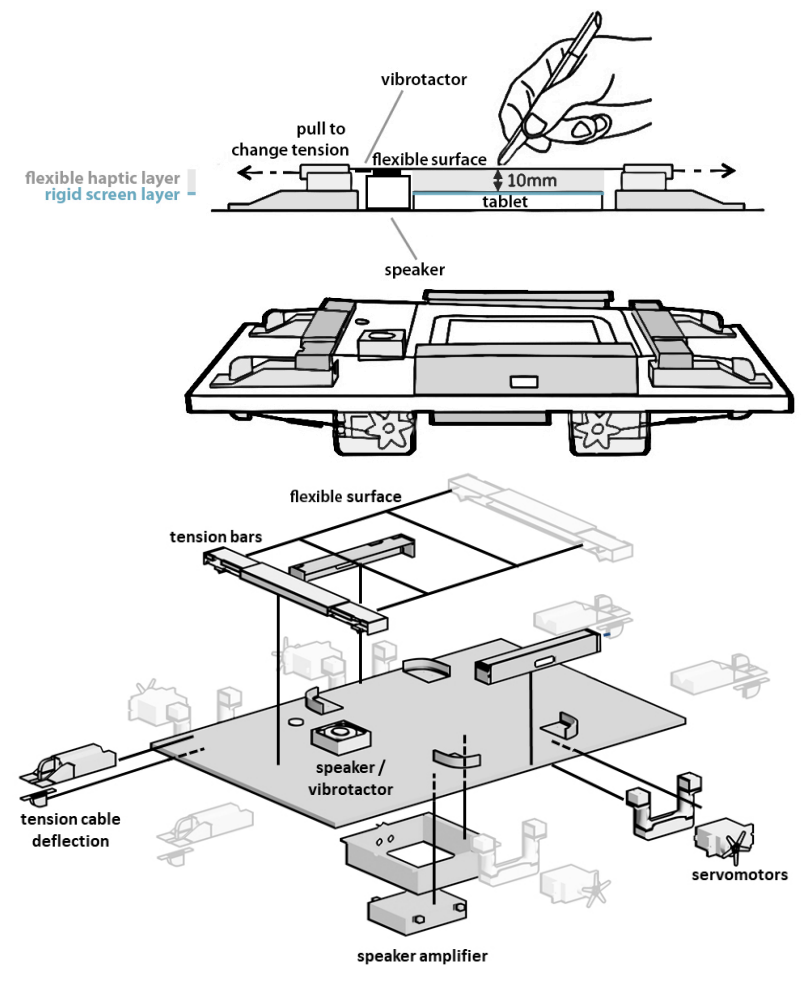

Figure 4. (Top) Schematic side view of the FleXurface system, showing the pulling mechanism to change the surface tension, (Bottom) explosion diagram of all system components. Transparent objects are further instances of the main "highlighted" functional components.

The gap between the flexible surface and the display is $10 \mathrm{~mm}$. In the studies, users looked almost straight down at the screen, limiting any potential parallax effects caused by this gap and we did not observe any negative effects. While parallax could be an issue for precise interaction with angled viewpoints, we can also point out that parallax effects are implicitly mitigated once the user presses the pen down towards the rigid display surface for direct manipulation of screen content. The gap between the flexible surface and the display is small enough to still enable us to track the pen reliably while it moves above the screen. Thus, the user can press the pen against the flexible surface without directly touching the screen and interact with the system.

The bars stretching the flexible surface are connected to four digital servos mounted underneath the surface onto which the tablet is mounted, through $0.5 \mathrm{~mm}$ nylon cables. The cables are tensioned between the bars and the digital servos by tiny adjustable spanners. The digital servos (Align DS655) are operated at $6 \mathrm{~V}$ over an external power supply that can drive the servos to their full extent at about $2 \mathrm{~A}$ per servo. The servos can theoretically move $60^{\circ}$ in $0.06 \mathrm{sec}$ but work slower under load (Table 1 ). The servos are currently 
operated in unison. The tension is defined by the servo angle and stretch. In comparison to the neutral position where the flexible surface is flat (at around $2 \mathrm{~mm}$ stretch, $65^{\circ}$ ), the surface can be stretched up to $12 \mathrm{~mm}$ (at $130^{\circ}$ servo angle). The "sweet spot" of the system is roughly between $2 \mathrm{~mm}$ and $11 \mathrm{~mm}$ stretch. To reduce the noise of the servos, which is audible during usage, we created a box-shaped "sound cover" with sound-damping material. This much reduces the higher frequency sounds of the servos (Fig. 5). The whole system was calibrated as described below.

The flexible haptic layer can be used in two modes: static or dynamic. When static, the servos build up surface tension before the pen enters the flexible haptic layer and retain the tension irrespective of user action. In contrast, dynamic tension is changed upon user events. In general, two kinds events can trigger tension changes in our system: pressing ("pushing a button") or sliding events ("moving over a GUI element"). Increasing the tension of the flexible material moves the pen away from the display, as the pen is forced upwards (Fig. 8B).

Tactile cues using membrane properties. Another noteworthy feature of the flexible material is that it serves as a membrane: as the surface is under sufficiently high tension, relayed vibrations can be sensed well when the pen is in contact with the swinging flexible material. Surprisingly, vibrations can even be sensed when the membrane is under low tension and touches the tablet screen. To explore the potential of such vibrotaction, we mounted a small speaker and a vibrotactor beside the tablet (Fig. 4). We connected the solid membrane of the loudspeaker to the flexible surface through a rubber ring on top of the speaker membrane. Thus, we can not only play audio, but have also the flexible surface function as an extended speaker membrane, even when it touches the tablet screen. Similar to previous work [44] and depending on the waveform, the flexible surface will vibrate (swing), providing tactile cues in unison with audio feedback, which can be sensed through the pen (see Table 1). As such, rendering tactile properties of textures is straightforward. The speaker is driven by a tiny $3 \mathrm{~W}$ mini amplifier and connected to the tablet line-out. This audio can normally be heard well, unless headphones are worn (during our experiments). For tactile feedback independent of audio, we employ a Precision Microdrives $12 \mathrm{~mm} / 3 \mathrm{~mm}$ pancake vibrotactor (with 1.3g). Note that the embedded loudspeaker of the tablet cannot directly provide vibrotactile feedback as it is not connected to the flexible material. Also, we can use the tablet vibrator when the system is used in "rigid mode" and pilot testing revealed that this vibration is perceived well, too.

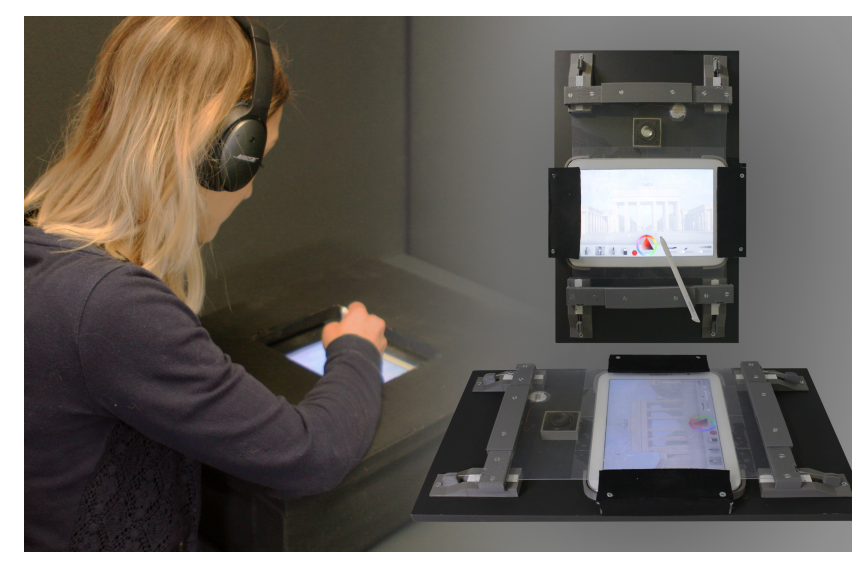

Figure 5. Apparatus with sound / visual cover (Left), top and side/top view of setup showing art application (Right).

\section{STUDY 1: FIRMNESS SIMULATION}

In our two user studies, we explored the potential of the haptic layer to convey different haptic properties that can, for example, be associated with different object materials, shapes or tools [47]. In study 1 , we looked closely at the perception of firmness, while study 2 investigated contour properties. Our haptic feedback was decoupled from visual feedback in our studies, to avoid this potentially confounding factor.

To characterize how well users can perceive and differentiate between different tensions of the flexible haptic layer, in study 1 we performed a just noticeable difference (JND) experiment, a regularly used task to assess how well users can differentiate between cues [34]. The following research question $(\mathrm{RQ})$ motivated us to explore this perceptual dimension:

\section{RQ1: What are the just noticeable differences (fNDs) in} firmness perception while pressing or sliding?

We performed two pilot studies. The first pilot was used to fine-tune the apparatus, identify technical issues, and test the parameters of the main study. The second pilot was a finger-based JND experiment with the same design as study 1. While we cannot track the finger through the flexible surface, we trained users to press the same distance down as in the main experiment (6 $\mathrm{mm}$ protrusion). Eight persons (2 females) aged 23-65 $(\mathrm{M}=35.4, \mathrm{SD}=16.4)$ participated in the pilot study. They had all used a pen-tablet sometimes $(75 \%)$ or frequently $(25 \%)$ before. For comparison, we also include these results in Fig. 6. The Weber fraction for finger sliding $(9 \%$, JND $0.31 \mathrm{~N})$ proved to be better than the pen. However, the press actions were far worse (24\%, JND $0.40 \mathrm{~N})$. This underlines why we chose to focus on peninstead of finger-based interaction. 
Apparatus. The apparatus was used in landscape mode (Fig. 5). Users wore noise-cancellation headphones playing white noise to remove any potential confounds from external audio cues. In addition to the sound cover, a second box was placed over the setup, which fully obscured the bars or vibrotactor movement. The outside of the box was covered with soft fabric, and users could rest their palm or arm on that cover.

Calibration. To calculate the pressure distribution, we performed multiple calibration steps. In a first step, we mounted a pressure sensor (Interlink 402) with a small disk similar to the pen-tip diameter in a mechanical contraption and measured the pressure at the five locations depicted in Fig. 3, at $6 \mathrm{~mm}$ protrusion depth. We then adjusted the tension on the servo cables such that positions $1 \& 4$, as well as $2 \& 5$ had comparable pressure profiles. As the display is not centered underneath the flexible material due to the need for further actuators (loudspeaker and vibrotactor), locations $1 \& 4$ and $2 \& 5$ produce slightly different forces (see Fig. 3). We measured pressure at the full tension range of the flexible material and calculated both the stress-strain curves and pressure accordingly. For this, we pressed the pressure sensor onto a scale, to correlate pressure to force. Then, we mounted the S-pen in the mechanical contraption and measured the pen-tip pressure again under the full tension range, at the middle position (number 3). The pentip was also matched with a force profile using the aforementioned method. Position 3 was used as main feedback area in our user studies. To avoid mis-calibration or bias, the top plastic layer was fixed during both calibration and studies.

Design. Study 1 followed a standard two alternative forced choice (2AFC) staircase protocol for calculating JNDs, where users had to compare two different tensions, stating if they felt different or the same [16,34]. The staircase protocol was used to identify at which threshold the users could still just feel a difference, by iteratively increasing or decreasing the tension around the threshold. Users performed the protocol for both pressing and sliding separately, as both are different variable compliant haptic events typical for interaction with FleXurface. Both pressing and sliding tasks were repeated two times. The base tension ("standard value") was chosen at $2 \mathrm{~mm}$ stretch $\left(65^{\circ}\right.$ servo angle), where the surface is flat. The staircase procedure started at $10 \mathrm{~mm}$ stretch $\left(110^{\circ}\right)$ and went down in $5^{\circ}$ steps, until no difference could be felt between the "standard" and "comparison" value. As can be seen in Fig. 3 , this step size produces fairly linear tension (N) differences. From then on, we used $1^{\circ}$ steps to adjust the simple up-down staircase procedure. The procedure was stopped after 50 steps, or 12 reversals. We chose $110^{\circ}$

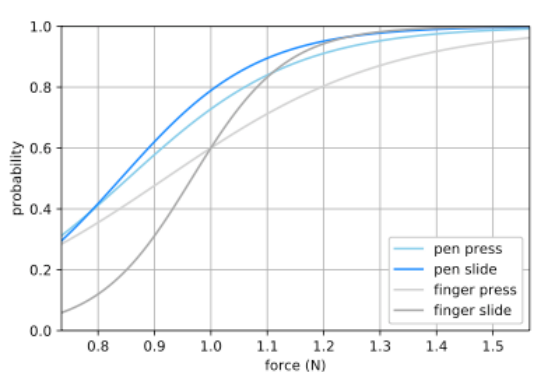

Figure 6. Psychometric curves (forces in N). Finger press/slide depict results of the pilot study.

$(1.63 \mathrm{~N})$ as the starting point, as it offers a tension clearly different from $65^{\circ}(0.74 \mathrm{~N})$. On the screen, a button was shown at position 3 (Fig. 3) that either had to be pressed, or slid over. For sliding, a marker was moved along the button that the user should follow, starting at $1.4 \mathrm{~cm}$ to the left of the button and ending $1.4 \mathrm{~cm}$ right of it. Pressing and sliding was performed at $6 \mathrm{~mm}$ surface protrusion. When the correct pen penetration depth was achieved, we indicated this through a color change (button or moving marker turning green). We implemented this feedback by reading the pen tip pressure and comparing it to the calibration measurements. During pressing tasks, users had to lift the pen completely from the surface between press actions. Between displaying different simulated buttons, the pressure was set to neutral before setting the tension again, to avoid potential visual cues based on the motion of the flexible surface. After pressing or sliding, users had to indicate if they could detect a difference or not, by pressing a button on the screen. After the study, a questionnaire recorded subjective ratings for the system, with a 5-point Likert scale.

Results and discussion. The sample was composed of 12 participants (one female), with an age range of 20-39 years and an average of $28.67(\mathrm{SD}=5.94)$. Most had normal vision, but 4 persons wore glasses or contact lenses. The majority had at least some experience with pen-based tablets, with 9 participants who used them sometimes $(75 \%)$ and 2 frequently (16.7\%). Subjects could differentiate well between tensions for press and slide actions. Based on the results of the experiment (see Fig. 6), we calculated the point of subjective equality and the JND threshold, according to [34], to define the Weber Fraction (WF). The average WFs were $16 \%$ for pen slide (JND $0.24 \mathrm{~N}$ ) and $19 \%$ for pen press (JND $0.28 \mathrm{~N}$ ). These results are in line with Jones et al. [34], who report firmness (stiffness) experiments that are in the $15-22 \%$ WF range. Intriguingly, participants could differentiate a bit better between tensions during sliding compared to pressing. Based on the JND results, we can support at least 4 differentiable firmness levels within the tension range of our current device. Note that during 


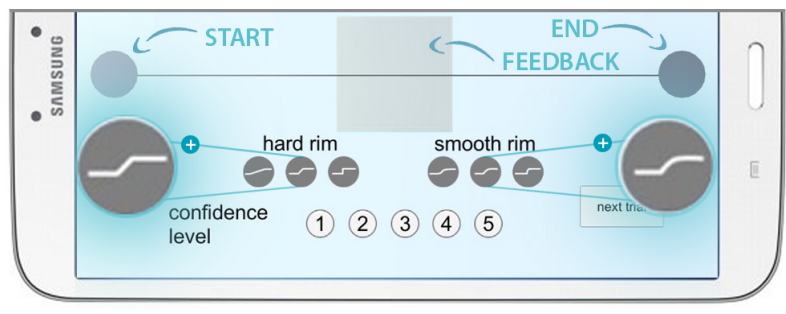

Figure 7. Contour experiment screen. Areas indicated by blue legends for illustrative purposes: start, feedback, and end areas at the top, and enlarged hard/smooth rim response buttons (with + sign) in the bottom half.

sliding, users perceived stimuli twice, when entering and exiting the button, which may have affected the outcome.

We report here only the most salient results from the questionnaire analysis. The results indicated that the system was easy to use and to learn for sliding $(\mathrm{M}=4.25$, $\mathrm{SD}=0.45$; resp. $\mathrm{M}=4.42, \mathrm{SD}=0.51$ ) and for pressing $(\mathrm{M}=4.58, \mathrm{SD}=0.51$; resp. $\mathrm{M}=4.5, \mathrm{SD}=0.67)$. Participants were neutral about the white noise sound they heard during the experiments $(\mathrm{M}=3.10, \mathrm{SD}=1.50)$ but stated that the servos could not be heard. Moreover, participants did not feel frustrated by the task (press $\mathrm{M}=3.58, \mathrm{SD}=1.31$; slide $\mathrm{M}=3.67, \mathrm{SD}=1.07$ ), felt confident operating the system (press $\mathrm{M}=4.08, \mathrm{SD}=0.79$; slide $\mathrm{M}=4.0, \mathrm{SD}=0.85$ ), and could sense the haptic cues well with the pen (press $\mathrm{M}=3.67, \mathrm{SD}=1.07$; slide $\mathrm{M}=3.58, \mathrm{SD}=0.9$ ). In general, ratings ranged from neutral to positive for both conditions.

What does the JND and variable compliant feedback range imply? Through the flexible surface we offer continuous variable compliant feedback that, in terms of forces, lies in the continuum between very soft mid-air feedback, such as provided by ultrasonic soundwaves [10], and the more firm, solenoid-based or vibrotactile feedback provided by non-tethered haptic pens, such as $[11,37,45,48]$. The feedback range can be best understood by the different sensations perceived when touching the human skin at the hand at different places. It depicts both softness and the capability to retake shape ("spring back") to its original shape. While requiring further investigation, we also assume the feedback, being variable compliant and soft, can potentially be associated with different kinds of materials, ranging from soft fabrics to rubber or silicone.

\section{STUDY 2: CONTOUR SIMULATION}

In our second study, we explored how the combination of the membrane functionality and flexibility of the material can be used to simulate contour aspects. Our study extends the initial exploration by Kim et al. [39] on using tactile cues to simulate rims on flat, rigid surfaces. We hypothesized that rim gradient perception decreases as a function of the speed of tension adjustment, where we expect that higher tension and shorter vibrotactile cues would lead to the perception of sharper rims, as vibration feedback adjusts softness perception [79].

RQ2: Do users attribute different combinations of tension change and vibrotactile cues to different object contour aspects, like shapes and gradients of button rims?

The study focused on the effect of different cue combinations (variable compliant, audio, vibration) on the resulting percept of different GUI button rim shapes and gradients. It employed a 3 (amount of tension change) $\mathrm{x} 3$ (speed of tension change) $\mathrm{x} 2$ (vibration durations) factorial design, resulting in 18 distinct combinations. The amount of tension change was $10^{\circ}$ ( $2 \mathrm{~mm}$ stretch), $30^{\circ}$ (6 mm stretch), or $50^{\circ}(10 \mathrm{~mm}$ stretch). Excluding the duration of the actual actuation, the speed of tension change was either "instant" (without additional delay) or was delayed by 100 or $200 \mathrm{~ms}$, while the vibration duration was either 150 or $250 \mathrm{~ms}$. The pancake vibration element provided the vibration pattern. For every combination, users had to judge both the gradient of the rim (one of 3 gradients, from almost flat to steep) and the shape of the rim, being either smooth (rounded) or sharp (edgy). Similar to study 1, users moved the pen along a line at a constant speed and we displayed the button contour options (but not the actual haptic button) during that action. After finishing each trial, participants had to select the perceived gradient and shape of the rim (forced choice, see Fig. 7). The study used the same setup as the first one. Results and discussion. 12 participants (5 female) took part, with ages ranging from 24 to 64 years $(M=35.25, S D$ $=13.55)$. One participant frequently used pen-tablets $(8.3 \%)$, seven sometimes (58.3\%), and four not at all (33.3\%). Perception of both sharpness and gradient aspects of button rims was affected by cue combinations, supporting shape recognition. However, such combinations have limits, as the kind of rim and gradient cannot always be easily separated. We used GEE analysis to examine the influence of the speed of the change in tension, amount of tension change, the vibration duration, and interactions on the shape choice. The choice between smooth and sharp rims was influenced by the amount (Wald's $\chi 2(2)=11.26$, $\mathrm{p}=.004)$ and the interaction of the amount and speed of tension change $\left(\chi^{2}(4)=19.24, \mathrm{p}=.001\right)$ but not by vibration duration $(\chi 2(1)=0.009, p=.923)$. For gradients, the analysis revealed a significant influence of the amount $(\chi 2(2)=23.13, p<.001)$ and speed of tension change $(\chi 2(2)=16.49, \mathrm{p}<.001)$ and the interaction of amount and speed $(\chi 2(4)=10.98, p=.027)$. Vibrotaction showed no significant effect. 


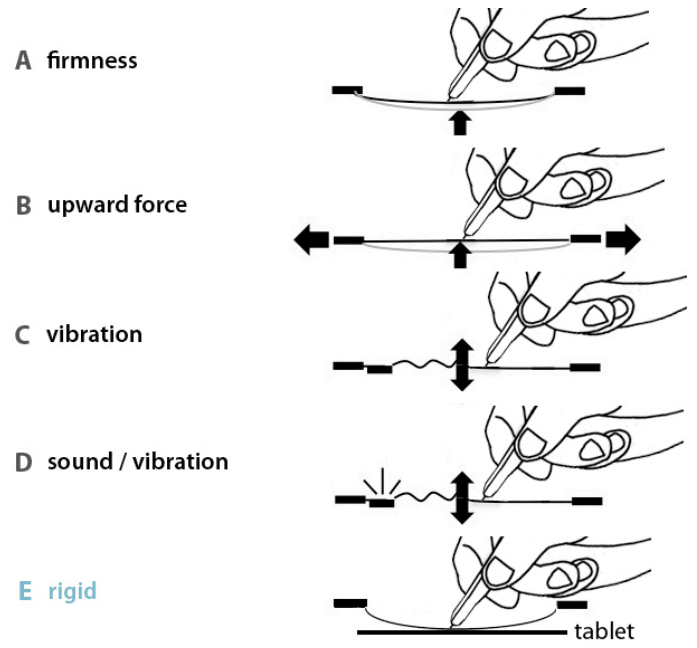

Figure 8. Feedback types afforded by our FleXurface system through different forms of actuation and the flexible (A-D) or rigid state (E) of the system.

We had initially hypothesized that rim gradient perception would decrease with slower tension speed adjustment. Conversely, higher tension and shorter vibrotactile cues would lead to the perception of sharper rims. Our results show that the perception of smooth rims with low gradients is enhanced by changing the tension only a little $(2 \mathrm{~mm}$ stretch), independent of the speed of change. The perception of sharp rims can be enhanced by a large tension change (10 $\mathrm{mm}$ stretch), which is further strengthened when this change occurs instantly. Medium gradients are perceived when applying medium and higher tension changes. The perception of a high gradient was enhanced only slightly with an instant high-range tension change (10 mm stretch).

The results from the questionnaires indicated that users had to concentrate on the task, in particular on the vibration. While this contrasts with previous work by Kim et al. [39], we point out that they coupled tactile cues to a rigid, not a flexible surface, and we attribute this result to the dampening inherent in a flexible surface.

We again report only the most salient insights for the analysis of the questionnaire data. Participants stated that their required concentration level was high for the rim study $(M=4.33, S D=1.15)$, which is in line with our expectations, as this perceptual task requires users to pay close attention. Some subjects also noted specifically that the multitasking nature of some tasks, i.e., the requirement to attend to different cues, was challenging. The mean confidence level for operating the system for all trials was neutral, $\mathrm{M}=3.09, \mathrm{SD}=0.73$. Although participants mostly agreed that the system was easy to use and to learn (mean ratings $\geq 4$ ), they did not agree that perceiving audio-tactile (press and slide) and vibrotactile cues (rim classification) was easy (press: $\mathrm{M}=2.0, \quad \mathrm{SD}=1.04 ;$ slide: $\mathrm{M}=2.17$, $\mathrm{SD}=1.34$, rim: $\mathrm{M}=2.17, \mathrm{SD}=1.19$ ). Consequently, ratings for the usefulness of the cues were also somewhat low. We expect that for easier tasks, ratings would be higher, as the pilots showed that, in principle, vibro- and audio-tactile can easily be noticed. Overall participants stated that they could receive cues through the pen well but felt neutral about rim classification.

What do the results of audio-tactile sensations imply? While the perception of the stimuli for rim detection requires concentration, the cues may prove useful for more general indications, such as hand guidance or display of warnings. We explore the potential for such indications in our art application.

\section{MULTILAYER INTERACTION DESIGN SPACE: CUES AND THEIR APPLICATION}

The FleXurface system does not only support the exploration of materials and spatial haptics that we use in real life $[40,47]$, but also the elasticity that simulates haptic aspects of, e.g., tools. Here, we will discuss how the pen can be actuated, and how actuation - including flexible and rigid state transitions - can be used within a sample art application. Finally, we discuss higher-level functionality.

How can the pen be actuated? Our system can provide different kinesthetic, tactile, and auditory cues (Fig. 8 A-E). Different levels of firmness can be simulated (A), which can be sensed by the user when pushing the pen downwards, until it touches the screen. The tension can also be changed suddenly, providing an upward force (B) that moves the pen away from the tablet. Due to the addition of the vibrotactor and speaker, vibration and combined sound/vibration cues are available (C, D). Alternatively, the variable compliant surface can be lowered so that the pen can operate directly on the rigid surface of the screen, while its motion is constrained on that 2D surface (E). These cues are comparable with cues provided in mobile devices, e.g., $[6,39,52,62,79]$, but are here directly coupled to the feedback provided through the flexible haptic layer.

Art application. We developed an art application (Fig. 9) to illustrate the capabilities of our system. The art application enables the user to draw and paint with different media (oil and watercolor painting with different brush types, drawing), by adjusting the "feeling" of the different tools (e.g., brush type versus pencil) and surface properties (e.g., paper roughness). Thus, the application actively uses the transition between flexible and rigid states. The user can experience the different media by adjusting the haptic simulation. We also explored a novel widget, namely a haptic slider. The slider allows the user to tune tool 
(brushes) and surface (paper roughness) settings, while immediately and directly feeling the effect. Upon a change of slider position, we directly map the value to the tool property being manipulated to the haptic layer and thus actuate the pen while it slides over the flexible surface. The flexible haptic layer plays a pivotal role in the application, by changing between the "fully flexible" state (simulating watercolor and oil paint brushes in our application) and the "rigid" state, where the flexible surface touches the rigid display of the tablet. The latter allows for a pen-on-paper feeling that is distinctively non-flexible. All tools actively make use of the pressure sensitive tip to change line/stroke thickness. Through vibration, we can simulate paper texture roughness during painting, by playing the sound and vibration associated with the tool-paper contact (using colored noise). Finally, we demonstrate an active form of guidance, through a painting-by-numbers example. This painting approach - often used by kids - has colored areas with strict boundaries, where paint is to be applied only within the borders. We provide guidance through the contour simulation method (study 2) to indicate when a boundary is being crossed. Early feedback from five users showed that the art application can be used well, and offers a more natural "feeling", in particular when using the paint tools. Also, the guidance method was found to be an interesting and useful aid.

What are the dependencies between the two layers? The two layers are directly dependent on each other during most interactions and afford transitions between flexible and rigid states. However, their dependency can differ. The flexible haptic layer is predominantly used for haptic feedback, while the rigid screen layer mainly serves for the display of visual content. Direct manipulation can occur

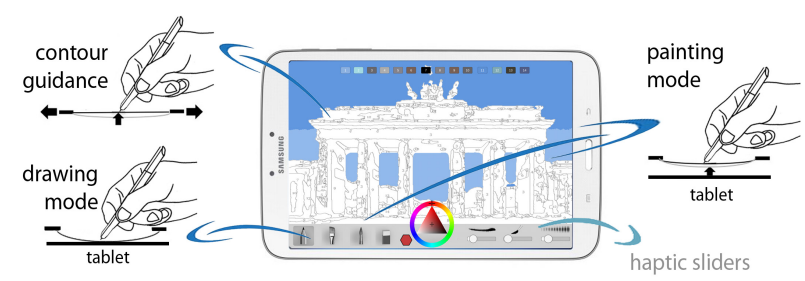

Figure 9. Art application: rigid and variable compliant (flexible) states simulate different tools. The paint-bynumbers application displays upward force to warn users when passing over a boundary of an area.

both in the haptic layer (pen pressure) or on the rigid layer (touch and pen pressure). Interaction with both layers can be performed serially, in parallel, or partly independent (Fig. 10). We expect that in most cases and before manipulating on-screen content, the user will, at least quickly, explore additional material or spatial haptic features of displayed content in the haptic layer, e.g., button properties, which corresponds to serial usage. On the other hand, both layers can be used in a parallel, coupled manner, for example when a tool is used continually, e.g., during painting, or when a widget such our haptic slider is used. Finally, the haptic layer can also be used partly independent of the tablet screen, e.g., when the system is used eyes-free $[8,36]$. Here, haptic features serve as the main interaction, independent of visual feedback and solely through pen interaction. In most cases the visual display serves as reference point for feedback in the flexible layer. As visual and haptic feedback are aligned, feedback will likely be mentally integrated, as predicted by the visual-haptic integration theory [76]. Yet, the flexible haptic layer can also serve as reference point for the visual display, guiding user actions. This might be useful to guide the user along serial

\begin{abstract}
interaction example
\end{abstract}

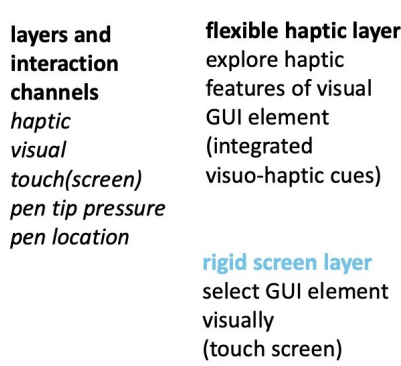
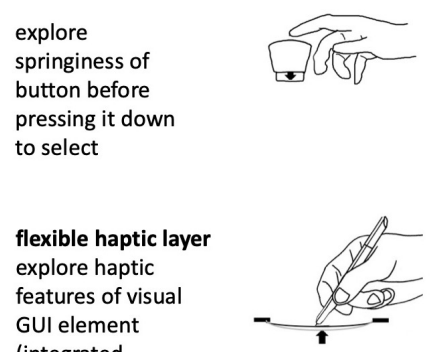

(touch screen)

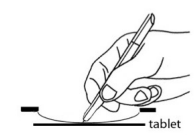

parallel

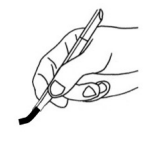
widths by changing pressure on (simulated) brush

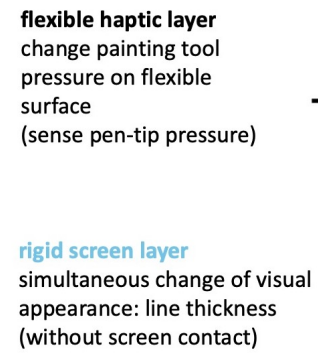
change painting tool pressure on flexible surface (sense pen-tip pressure)

rigid screen layer simultaneous change of visual appearance: line thickness (without screen contact)

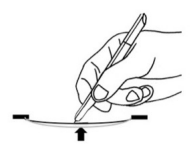

partly independent

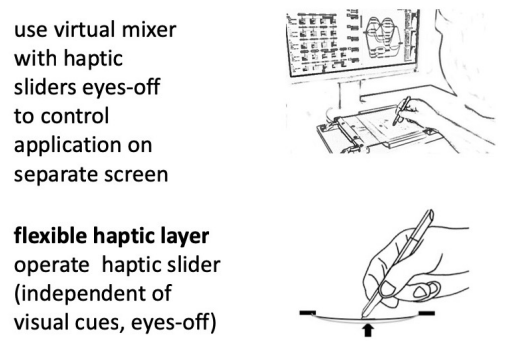

rigid screen layer

track pen location only

Figure 10. Multilayer dependencies: transitions between flexible and rigid states / layers in different kinds of haptic-feedback supported tasks. Note that in the partly independent task visual feedback is left out deliberately, to support eyes-off operation. Still, in many application scenarios, the sliders would also be visualized. 
Table 1 - Non-visual cues and their characteristics. Average latencies were measured at all 5 display locations (Fig. 3). Latencies exclude the pen latency, which is about $95 \mathrm{~ms}$.

\begin{tabular}{|c|c|c|c|c|c|c|c|}
\hline Actuator & Actuation & Latency & $\begin{array}{l}\text { Pen } \\
\text { actuation }\end{array}$ & Cue Perception & $\begin{array}{l}\text { Widget, tools, } \\
\text { surfaces, guidance }\end{array}$ & $\begin{array}{l}\text { Studies / } \\
\text { use case }\end{array}$ & $\begin{array}{l}\text { Related } \\
\text { work }\end{array}$ \\
\hline $\begin{array}{l}\text { Flexible } \\
\text { surface }\end{array}$ & $\begin{array}{l}\text { Tension } \\
\text { change }\end{array}$ & $\begin{array}{l}70-185 \mathrm{~ms} \text {, } \\
\text { from low to } \\
\text { high tension } \\
\text { (from neutral } \\
\text { position) }\end{array}$ & $\begin{array}{l}\text { Resist } \\
\text { downward } \\
\text { motion } \\
\text { toward screen }\end{array}$ & $\begin{array}{l}\text { Variable compliant } \\
\text { haptics: surface } \\
\text { firmness } \\
\text { (kinesthetic) }\end{array}$ & $\begin{array}{l}\text { Rigid versus soft or } \\
\text { springy buttons, art } \\
\text { tools, surface } \\
\text { properties }\end{array}$ & $\begin{array}{l}\text { Study } 1, \\
\text { art app }\end{array}$ & $\begin{array}{l}{[20,49,67,} \\
68,71]\end{array}$ \\
\hline \multirow[t]{2}{*}{ Speaker } & $\begin{array}{l}\text { Vibrate } \\
\text { variable } \\
\text { compliant } \\
\text { surface }\end{array}$ & $\begin{array}{l}\approx 47 \mathrm{~ms}, \text { all } \\
\text { positions }\end{array}$ & Vibration & $\begin{array}{l}\text { Vibration } \\
\text { (tactile) }\end{array}$ & \multirow[t]{3}{*}{$\begin{array}{l}\text { Paper textures, } \\
\text { button surfaces, } \\
\text { warnings, guidance, } \\
\text { selection cues }\end{array}$} & \multirow[t]{3}{*}{$\begin{array}{l}\text { Study } 2, \\
\text { art app }\end{array}$} & $\begin{array}{l}{[6,29,39,5} \\
1,52,59,62, \\
79]\end{array}$ \\
\hline & Audio & N/A & & Sound (auditory) & & & {$[30,77]$} \\
\hline $\begin{array}{l}\text { Vibro- } \\
\text { tactor }\end{array}$ & Vibration & $\begin{array}{l}\approx 24 \mathrm{~ms} \text {, all } \\
\text { positions }\end{array}$ & Vibration & $\begin{array}{l}\text { Vibration } \\
\text { (tactile) }\end{array}$ & & & \\
\hline $\begin{array}{l}\text { Tablet } \\
\text { screen }\end{array}$ & Visual & $\mathrm{N} / \mathrm{A}$ & $\begin{array}{l}\text { Passive: } \\
\text { Restrict } \\
\text { motion }\end{array}$ & $\begin{array}{l}\text { Constrained motion } \\
\text { (kinesthetic) }\end{array}$ & $\begin{array}{l}\text { Rigid instead of soft } \\
\text { art tools }\end{array}$ & Art app & \\
\hline
\end{tabular}

the spatial layout shown on the visual display, e.g., while moving among different menu structures. Finally, while the rigid screen layer is mostly used for visual feedback and direct manipulation, it can constrain the interaction volume.

What is the higher-level functionality afforded by the system and how can an interface designer use it? The art application illustrates how we can map pen actuation to functionality. Here, we will discuss the higher-level affordances of our FleXurface system, as summarized in Table 1. Based on the material properties of our flexible surface, haptic exploration is bound to the simulation of soft deformable materials, not unlike [60], yet more controllable. Even more so, the coupling of both (a) the speaker and vibrotactor and (b) the rigid surface of the tablet screen affords a number of cues that map well to application requirements. Thereby, feedback of our flexible haptic layer resides in the continuum between the very light mid-air feedback, such as provided through ultrasound feedback [10], and the firmer haptic feedback associated with rigid display interaction, e.g., via haptic pens. Our type of variable compliant haptic feedback superficially resembles earlier work by Chu et al [14], who used a haptic mouse wheel to communicate conviction using force, where greater conviction requires greater force.

Interface designers can take advantage of the variable compliant feedback to enhance widget interaction, such as buttons and sliders. Selection of widgets is supported by measuring pen-tip pressure, or by pressing the pen down to touch the rigid surface. The different levels of softness could be used to design menu systems with different functional groupings, e.g., by adding to or complementing visual groupings [7]. Stiffer feedback could also be used to make potentially harmful delete actions harder to perform. On the other hand, physical button contours can be used to support eyes-off operation of a system. Although the system is not mobile yet, our flexible haptic feedback functionality could, e.g., be used to support control of a cellphone in the users pocket, where audio and vibration could be used to provide selection cues ("clicks" [31]). Such properties would be explored by the user by serially interacting with both layers. The flexible haptic layer can also support novel widgets. In our art application, we show a haptic slider, where tool or surface changes can be experienced immediately during sliding. Interface designers could use such sliders in cases where direct feedback about the effect of a change selected through a slider is important.

Tactile or kinesthetic cues enhance the operation of tools that exhibit "elastic" properties. Such cues can enhance the experience and potentially also performance with such tools $[4,75]$, as these cues provide indications of the realworld haptic properties that help the user understand how the tool performs while using it. We highlighted the potential of such cues in our art application, where the experience of different brush properties subjectively enhanced interaction.

Our system can also render other kinds of surface properties, through firmness and audio-tactile feedback. Different textures could be useful in the design of novel menu systems where not only color but also texture is used to highlight functional groupings. Other application domains include surface simulation in simulation systems, e.g., to simulate skin, while the addition of other surface characteristics, e.g., to simulate friction, is a potential area for future follow up. 
Finally, the system can be used for guidance. Simulated rims [39] or textures, but also other sudden changes of feedback, such as an abrupt upward force, can be used to display a warning or to guide hand motion. As an example, by moving the pen upwards, users could be warned about potentially harmful implications, e.g., on a "delete all" button.

\section{LIMITATIONS}

While FleXurface supports a large set of haptic cues, it still only supports a subset of all possible haptic features. For example, weight, temperature and volume are not supported in the current system. Temperature feedback could be quite easily integrated into the pen using a Peltier element. But weight could be challenging to simulate without tethering. Furthermore, while the contour simulation mostly depends on rims, it lacks the volumetric information that can also be used for contour detection. Moreover, FleXurface can simulate only a limited range of variable compliant stiffness (roughly $0.7 \mathrm{~N}$ to $1.6 \mathrm{~N}$ ). With the current technical design, it would be difficult to simulate much firmer materials, as the servos will not be able to actuate such a material. Also, the current surface material does not support direct finger interaction, as it blocks capacitive sensing. Other flexible surface materials could be used to simulate different ranges of softer materials, as long as the material is transparent. Perceiving textures through the flexible surface material likely has some limitations as the material absorbs vibration differently under different tensions (especially when it touches the rigid display), which warrants further study. Finally, while the sound of the servos could not be heard during experiments due to the headphones, it might have to be blocked by other means for situations where headphones are not an option.

The pen latency of the current implementation is also a potential issue but can be improved by using more recent systems that have lower latency, e.g., the Microsoft surface. As we noted before, to avoid this potentially confounding factor, our haptic feedback was decoupled from the visual feedback in our studies. Still, the latency was rarely noticed in our art application, as painting movements were rather deliberate and slow. Furthermore, there is no servo latency for continuous static feedback, e.g., while using a brush. This may be similar to other haptic exploration tasks, such as pressure sensing and contour exploration, which are typically performed more slowly [46]. Finally, while our users experienced cognitive load, we could not yet clearly identify the source. As such, it is an issue that warrants further research.

\section{CONCLUSION AND FUTURE WORK}

In this paper, we presented a multilayer feedback approach for pen-based tablet interaction. To our knowledge,
FleXurface is the first system that demonstrates combined kinesthetic (variable compliant), tactile, and rigid feedback in a unified system for pen-based tablet interaction. Most other systems simulate only single haptic properties, in part through sensory substitution. For pen-based technologies, haptic feedback tends to be hard to achieve without tethering the pen, a problem we overcome with FleXurface. Overall, we report on the following contributions:

(A) Multilayer haptic interaction approach: We presented a novel haptic pen-based tablet interaction approach that affords the exploration of haptic features, e.g., firmness, contours, and texture, through a flexible haptic and a rigid screen layer. The user can seamlessly transition between flexible and rigid states with the untethered pen. We showed how cues can be applied and combined to elicit different widget, tool and surface properties, and also for guidance.

(B) Flexible haptic layer properties: We characterized the perceptual properties of the flexible haptic layer through two user studies that investigate different haptic features. In our first study, we performed a JND experiment to assess how well users can perceive tension (firmness) differences. The identified JND for pressing and sliding identify four differentiable tension (firmness) levels for our system. In our second study we explored how the membrane properties of the flexible haptic layer can display shape cues (rim/contour aspects). We showed that a higher tension range and velocity can simulate sharper and steeper button rims, while lower tension was perceived as smoother and flatter rims.

(C) Application: Through an art application, we showed how the different feedback possibilities afforded by the multilayer approach of FleXurface can enhance interaction, illustrating the potential of the system.

What are future directions? The art application is a good example of the different ranges of feedback that FleXurface supports. We assume that similar feedback mechanisms can also be deployed in other contexts. For example, accessibility might be increased by our extended widget feedback and guidance mechanisms, as FleXurface may ease interaction when operating under constrained visual conditions. Furthermore, we believe that the system can also be used in applications that simulate soft variable compliant material, including, but not limited to, medical applications that deal with skin. These directions are an area of future work.

Previous work has also shown that tactile cues can improve performance with widgets [6,15,21,51], similar to how haptic feedback is used in machinery design [8]. As we do not have to substitute haptics (kinesthetic cues) through 
tactile feedback, we assume that our multilayer approach can further enhance performance for such tasks and support higher accuracy. Though previous work shows initial positive indications [20], this latter aspect deserves further study, to show to what extent variable compliant haptic feedback is better than vibration-only feedback. Furthermore, previous work [42] indicated that haptic firmness discrimination performance could be improved when visual feedback is provided along with haptics, which is another venue for further study. While haptic cues work within the non-visual sensory channel, they also afford eyes-off interaction, another area that deserves follow-up. In addition, it will be interesting to assess to what extend the system can improve expressiveness [4,75].

While the current setup has mainly been used to explore the design space of our multilayer approach, miniaturization would be necessary to use our system in the mobile domain. A potential solution is the usage of deflection rollers to wrap the material more effectively around the display with smaller servos. Another option is to use quieter linear actuators. An additional direction we are currently following up is to lower the system into a table, making the whole setup a tabletop system where the hands can rest on the table surface. Finally, while the pen interaction has shown good performance, finger input did not always produce satisfactory results in our pilot JND study, especially for press actions. Yet, finger input is clearly an interesting direction to explore, as sliding produced satisfactory results. To support finger interaction, other flexible materials, such as those that include graphite particles, could be used.

\section{REFERENCES}

[1] Motoyuki Akamatsu and Sigeru Sato. 1994. A multi-modal mouse with tactile and force feedback. International Journal of HumanComputer Studies 40, 3: 443-453. https://doi.org/10.1006/ijhc.1994.1020

[2] Michelle Annett, Tovi Grossman, Daniel Wigdor, and George Fitzmaurice. 2011. Medusa: a proximity-aware multi-touch tabletop. In Proceedings of the 24th annual ACM symposium on User interface software and technology - UIST '11, 337. https://doi.org/10.1145/2047196.2047240

[3] Lukas Batteau, Alan Liu, Josephus Maintz, Yogendra Bhasin, and Mark Bowyer. 2004. A Study on the Perception of Haptics in Surgical Simulation. Springer Lecture Notes in Computer Science 3078: 185192. https://doi.org/10.1007/b98155

[4] Bill Baxter, Vincent Scheib, Ming C. Lin, and Dinesh Manocha. 2001. DAB: interactive haptic painting with $3 \mathrm{D}$ virtual brushes. In Proceedings of the 28th annual conference on Computer graphics and interactive techniques - SIGGRAPH '01, 461-468. https://doi.org/10.1145/383259.383313

[5] Hrvoje Benko, Christian Holz, Mike Sinclair, and Eyal Ofek. 2016. NormalTouch and TextureTouch: High-fidelity 3D Haptic Shape Rendering on Handheld Virtual Reality Controllers. In Proceedings of the 29th Annual Symposium on User Interface Software and Technology - UIST '16, 717-728. https://doi.org/10.1145/2984511.2984526

[6] Stephen Brewster, Faraz Chohan, and Lorna Brown. 2007. Tactile feedback for mobile interactions. In Proceedings of the SIGCHI conference on Human factors in computing systems - CHI '07, 159. https://doi.org/10.1145/1240624.1240649

[7] Duncan Brumby and Susan Zhuang. 2015. Visual Grouping in Menu Interfaces. In Proceedings of the 33rd Annual ACM Conference on Human Factors in Computing Systems - CHI '15, 4203-4206. https://doi.org/10.1145/2702123.2702177

[8] Hans-Jorg Bullinger, Peter Kern, and Martin Braun. 1997. Controls. In Handbook of Human Factors and Ergonomics, Gavriel Salvendy (ed.). John Wiley \& Sons.

[9] Juan Carrascal and Roel Vertegaal. 2017. Effects of Tactile Feedback on the Perception of Virtual Shapes on Non-Planar Display Objects. In ACM Conference on Human Factors in Computing Systems (CHI '2017), 4417-4423.

[10] Tom Carter, Sue Ann Seah, Benjamin Long, Bruce Drinkwater, and Sriram Subramanian. 2013. UltraHaptics: multi-point mid-air haptic feedback for touch surfaces. In Proceedings of the 26th annual ACM symposium on User interface software and technology - UIST '13, 505-514. https://doi.org/10.1145/2501988.2502018

[11] Dapeng Chen, Aiguo Song, and Lei Tian. 2015. A novel miniature multi-mode haptic pen for image interaction on mobile terminal. In 2015 IEEE International Symposium on Haptic, Audio and Visual Environments and Games (HAVE), 1-6. https://doi.org/10.1109/HAVE.2015.7359445

[12] Victor Cheung, Jens Heydekorn, Stacey Scott, and Raimund Dachselt 2012. Revisiting hovering: interaction guides for interactive surfaces. In Proceedings of the $2012 \mathrm{ACM}$ international conference on Interactive tabletops and surfaces - ITS '12, 355. https://doi.org/10.1145/2396636.2396699

[13] Youngjun Cho, Andrea Bianchi, Nicolai Marquardt, and Nadia Bianchi-Berthouze. 2016. RealPen: Providing Realism in Handwriting Tasks on Touch Surfaces using Auditory-Tactile Feedback. In Proceedings of the 29th Annual Symposium on User Interface Software and Technology - UIST '16, 195-205. https://doi.org/10.1145/2984511.2984550

[14] Gerry Chu, Tomer Moscovich, and Ravin Balakrishnan. 2009. Haptic conviction widgets. In Proceedings of Graphics Interface 2009, 207210.

[15] Andy Cockburn and Stephen Brewster. 2005. Multimodal feedback for the acquisition of small targets. Ergonomics 48, 9: 1129-50. https://doi.org/10.1080/00140130500197260

[16] Tom Cornsweet. 1962. The staircrase-method in psychophysics. The American journal of psychology 75: 485-91. Retrieved March 9, 2017 from http://www.ncbi.nlm.nih.gov/pubmed/13881416

[17] Heather Culbertson and Katherine J. Kuchenbecker. 2017. Ungrounded Haptic Augmented Reality System for Displaying Roughness and Friction. IEEE/ASME Transactions on Mechatronics 22, 4: 1839-1849. https://doi.org/10.1109/TMECH.2017.2700467

[18] Nicholas Fellion, Thomas Pietrzak, and Audrey Girouard. 2017. FlexStylus: Leveraging Bend Input for Pen Interaction. In Proceedings of the 30th Annual ACM Symposium on User Interface Software and Technology - UIST '17, 375-385. https://doi.org/10.1145/3126594.3126597

[19] Julien Fleureau, Yoan Lefevre, Fabien Danieau, Philippe Guillotel, and Antoine Costes. 2016. Texture Rendering on a Tactile Surface Using Extended Elastic Images and Example-Based Audio Cues. Springer, Cham, 350-359. https://doi.org/10.1007/978-3-319-42321032

[20] Sean Follmer, Daniel Leithinger, Alex Olwal, Nadia Cheng, and Hiroshi Ishii. 2012. Jamming user interfaces: programmable particle stiffness and sensing for malleable and shape-changing devices. In Proceedings of the 25th annual ACM symposium on User interface software and technology - UIST '12, 519. https://doi.org/10.1145/2380116.2380181

[21] Masaaki Fukumoto and Toshiaki Sugimura. 2001. Active click: tactile feedback for touch pannels. In CHI '01 extended abstracts on Human factors in computing systems - CHI '01, 121. https://doi.org/10.1145/634067.634141

[22] David Gallant, Andrew Seniuk, and Roel Vertegaal. 2008. Towards more paper-like input: flexible input devices for foldable interaction styles. In Proceedings of the 21st annual ACM symposium on User interface software and technology - UIST '08, 283. https://doi.org/10.1145/1449715.1449762 
[23] George Gescheider, William Barton, Michael Bruce, Jeffrey Goldberg, and Michael Greenspan. 1969. Effects of simultaneous auditory stimulation on the detection of tactile stimuli. Journal of Experimental Psychology 81, 1: 120-125. https://doi.org/10.1037/h0027438

[24] Ole-Christoffer Grønmo, John Oommen, Svein Myrer, Morten Olsen. 2007. Learning automata-based solutions to the nonlinear fractional knapsack problem with applications to optimal resource allocation. IEEE Transactions on Systems, Man and Cybernetics 37, 1: 166-175.

[25] Sidhant Gupta, Tim Campbell, Jeffrey Hightower, and Shwetak Patel. 2010. Squeeze- Block: using virtual springs in mobile devices for eyes-free interaction. In Proceedings of the 23nd annual ACM symposium on User interface software and technology - UIST ' 10 , 101. https://doi.org/10.1145/1866029.1866046

[26] Martin Hachet and Alexander Kulik. 2008. Elastic Control for Navigation Tasks on Pen-based Handheld Computers. In 2008 IEEE Symposium on 3D User Interfaces, 91-96. https://doi.org/10.1109/3DUI.2008.4476597

[27] Moustapha Hafez. 2007. Tactile interfaces: technologies, applications and challenges. The Visual Computer 23, 4: 267-272. https://doi.org/10.1007/s00371-007-0102-2

[28] Jaehyun Han, Jiseong Gu, and Geehyuk Lee. 2014. Trampoline: a double-sided elastic touch device for creating reliefs. In Proceedings of the 27th annual ACM symposium on User interface software and technology - $\quad$ UIST '14, 383-388, https://doi.org/10.1145/2642918.2647381

[29] Chris Harrison and Scott Hudson. 2009. Texture displays: a passive approach to tactile presentation. In Proceedings of the 27th international conference on Human factors in computing systems CHI 09, 2261. https://doi.org/10.1145/1518701.1519047

[30] Eve Hoggan, Andrew Crossan, Stephen Brewster, and Topi Kaaresoja. 2009. Audio or tactile feedback: which modality when? In Proceedings of the 27th international conference on Human factors in computing systems - CHI 09, 2253. https://doi.org/10.1145/1518701.1519045

[31] Eve Hoggan, Topi Kaaresoja, Pauli Laitinen, and Stephen Brewster. 2008. Crossmodal congruence: the look, feel and sound of touchscreen widgets. In Proceedings of the 10th international conference on Multimodal interfaces - IMCI '08, 157 https://doi.org/10.1145/1452392.1452423

[32] David Holman and Roel Vertegaal. 2008. Organic user interfaces: designing computers in any way, shape, or form. Communications of the ACM 51, 6: 48. https://doi.org/10.1145/1349026.1349037

[33] Yvonne Jansen, Thorsten Karrer, and Jan Borchers. 2010. MudPad: tactile feedback and haptic texture overlay for touch surfaces. In ACM International Conference on Interactive Tabletops and Surfaces - ITS '10, 11. https://doi.org/10.1145/1936652.1936655

[34] Lynette Jones and Hong Tan. 2013. Application of Psychophysical Techniques to Haptic Research. IEEE Transactions on Haptics 6, 3: 268-284. https://doi.org/10.1109/TOH.2012.74

[35] Kurt Kaczmarek, John Webster, Paul Bach-y-Rita, and Willis Tompkins. 1991. Electrotactile and vibrotactile displays for sensory substitution systems. IEEE transactions on bio-medical engineering 38, 1: 1-16. https://doi.org/10.1109/10.68204

[36] Raine Kajastila and Tapio Lokki. 2009. A gesture-based and eyes-free control method for mobile devices. In Proceedings of the 27th international conference extended abstracts on Human factors in

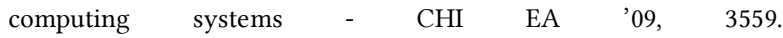
https://doi.org/10.1145/1520340.1520534

[37] Sho Kamuro, Kouta Minamizawa, Naoki Kawakami, and Susumu Tachi. 2009. Ungrounded kinesthetic pen for haptic interaction with virtual environments. In RO-MAN 2009 - The 18th IEEE International Symposium on Robot and Human Interactive Communication, 436441. https://doi.org/10.1109/ROMAN.2009.5326217

[38] Johan Kildal. 2010. 3D-press: haptic illusion of compliance when pressing on a rigid surface. In International Conference on Multimodal Interfaces and the Workshop on Machine Learning for Multimodal Interaction on - ICMI-MLMI '10, 1 https://doi.org/10.1145/1891903.1891931

[39] Seung-Chan Kim, Ali Israr, and Ivan Poupyrev. 2013. Tactile rendering of $3 \mathrm{D}$ features on touch surfaces. In Proceedings of the 26th annual ACM symposium on User interface software and technology - UIST '13, 531-538. https://doi.org/10.1145/2501988.2502020

[40] Roberta Klatzky, Dianne Pawluk, and Angelika Peer. 2013. Haptic Perception of Material Properties and Implications for Applications. Proceedings of the IEEE PP, 99: 1-12. https://doi.org/10.1109/JPROC.2013.2248691

[41] Roberta Klatzky and Susan Lederman. 2010. Multisensory Texture Perception. In Multisensory Object Perception in the Primate Brain. Springer New York, New York, NY, 211-230 https://doi.org/10.1007/978-1-4419-5615-6_12

[42] Maria Korman, Kinneret Teodorescu, Adi Cohen, Miriam Reiner, and Daniel Gopher. 2012. Effects of Order and Sensory Modality in Stiffness Perception. Presence: Teleoperators and Virtual Environments 21, 3: 295-304. https://doi.org/10.1162/PRES_a_00114

[43] Ernst Kruijff and Aaeldrik Pander. 2005. Experiences of using Shockwaves for Haptic Sensations. In Proceedings of 3D user interface workshop, IEEE Virtual Reality Conference (VR2005).

[44] Ernst Kruijff, Gerold Wesche, Kai Riege, Gernot Goebbels, Martijn Kunstman, and Dieter Schmalstieg. 2006. Tactylus, a Pen-Input Device exploring Audiotactile Sensory Binding. In Proceedings of the ACM Symposium on Virtual Reality Software \& Technology 2006 (VRST 2006)

[45] Ki-Uk Kyung and Jun-Young Lee. 2008. wUbi-Pen: windows graphical user interface interacting with haptic feedback stylus. In ACM SIGGRAPH 2008 new tech demos on - SIGGRAPH '08, 1-4. https://doi.org/10.1145/1401615.1401657

[46] Susan Lederman and Roberta Klatzky. 1993. Extracting object properties through haptic exploration. Acta Psychologica 84, 1: 2940. https://doi.org/10.1016/0001-6918(93)90070-8

[47] Susan Lederman and Roberta Klatzky. 1987. Hand movements: A window into haptic object recognition. Cognitive Psychology 19, 3: 342-368. https://doi.org/10.1016/0010-0285(87)90008-9

[48] Johnny C. Lee, Paul Dietz, Darren Leigh, William Yerazunis, and Scott Hudson. 2004. Haptic pen: a tactile feedback stylus for touch screens. In Proceedings of the 17th annual ACM symposium on User interface software and technology - UIST '04, 291. https://doi.org/10.1145/1029632.1029682

[49] Sang-Su Lee, Sohyun Kim, Bipil Jin, Eunji Choi, Boa Kim, Xu Jia, Daeeop Kim, and Kun-pyo Lee. 2010. How users manipulate deformable displays as input devices. In Proceedings of the 28th international conference on Human factors in computing systems CHI '10, 1647. https://doi.org/10.1145/1753326.1753572

[50] Charles Lenay, Olivier Gapenne, Sylvain Hanneton, Catherine Genouëlle, and Christelle Marque. 2003. Sensory Substitution: Limits and Perspectives. In Touching for Knowing, Y Hatwell, A Streri and E Gentaz (eds.). 275-292.

[51] Rock Leung, Karon MacLean, Martin Bue Bertelsen, and Mayukh Saubhasik. 2007. Evaluation of haptically augmented touchscreen gui elements under cognitive load. In Proceedings of the ninth international conference on Multimodal interfaces - ICMI '07, 374. https://doi.org/10.1145/1322192.1322258

[52] Vincent Levesque, Louise Oram, Karon MacLean, Andy Cockburn, Nicholas Marchuk, Dan Johnson, J. Edward Colgate, and Michael Peshkin. 2011. Frictional widgets: enhancing touch interfaces with programmable friction. In Proceedings of the 2011 annual conference extended abstracts on Human factors in computing systems - CHI EA '11, 1153. https://doi.org/10.1145/1979742.1979713

[53] Liping Lin, Yongtian Wang, Yue Liu, and Makoto Sato. 2011. Application of Pen-Based Planar Haptic Interface in Physics Education. In 2011 12th International Conference on ComputerAided Design and Computer Graphics, 375-378. https://doi.org/10.1109/CAD/Graphics.2011.53

[54] Paul Lubos, Oscar Ariza, Gerd Bruder, Florian Daiber, Frank Steinicke, and Antonio Krüger. 2015. HoverSpace. Springer International Publishing, 259-277. https://doi.org/10.1007/978-3-31922698-9_17

[55] Alexander Marquardt, Ernst Kruijff, Christina Trepkowski, Jens Maiero, Andrea Schwandt, André Hinkenjann, Wolfgang Stuerzlinger, and Johannes Schöning. 2018. Audio-tactile proximity feedback for enhancing 3D manipulation. In Proceedings of the 24th ACM Symposium on Virtual Reality Software and Technology VRST '18, 1-10. https://doi.org/10.1145/3281505.3281525 
[56] Alexander Marquardt, Jens Maiero, Ernst Kruijff, Christina Trepkowski, Andrea Schwandt, André Hinkenjann, Johannes Schöning, and Wolfgang Stuerzlinger. 2018. Tactile hand motion and pose guidance for 3D interaction. In Proceedings of the 24th ACM Symposium on Virtual Reality Software and Technology - VRST '18, 1-10. https://doi.org/10.1145/3281505.3281526

[57] Nicolai Marquardt, Miguel Nacenta, James Young, Sheelagh Carpendale, Saul Greenberg, and Ehud Sharlin. 2009. The Haptic Tabletop Puck: tactile feedback for interactive tabletops. In Proceedings of the ACM International Conference on Interactive Tabletops and Surfaces - ITS '09, 85. https://doi.org/10.1145/1731903.1731922

[58] Laurent Mignonneau and Christa Sommerer. 2005. Nano-Scape: Experiencing Aspects of Nanotechnology through a Magnetic ForceFeedback Interface. In Proceedings of the ACM SIGCHI International Conference on Advances in Computer Entertainment Technology.

[59] Joe Mullenbach, Craig Shultz, Anne Marie Piper, Michael Peshkin, and J. Edward Colgate. 2013. Surface haptic interactions with a TPad tablet. In Proceedings of the adjunct publication of the 26th annual ACM symposium on User interface software and technology - UIST '13 Adjunct, 7-8. https://doi.org/10.1145/2508468.2514929

[60] Mathias Müller, Anja Knöfel, Thomas Gründer, Ingmar Franke, and Rainer Groh. 2014. FlexiWall: Exploring Layered Data with Elastic Displays. In Proceedings of the Ninth ACM International Conference on Interactive Tabletops and Surfaces - ITS '14, 439-442. https://doi.org/10.1145/2669485.2669529

[61] Joshua Peschke, Fabian Göbel, Thomas Gründer, Mandy Keck, Dietrich Kammer, and Rainer Groh. 2012. DepthTouch. In Proceedings of the International Working Conference on Advanced $\begin{array}{lllll}\text { Visual Interfaces } & - & \text { AVI } & \text { '12, } & 770 .\end{array}$ https://doi.org/10.1145/2254556.2254706

[62] Ivan Poupyrev and Shigeaki Maruyama. 2002. Drawing with feeling: designing tactile display for pen. In ACM SIGGRAPH 2002 conference abstracts and applications on - SIGGRAPH '02, 173. https://doi.org/10.1145/1242073.1242186

[63] Ivan Poupyrev, Makoto Okabe, and Shigeaki Maruyama. 2004. Haptic feedback for pen computing: directions and strategies. In Extended abstracts of the 2004 conference on Human factors and computing systems - CHI '04, 1309. https://doi.org/10.1145/985921.986051

[64] Dmitry Pyryeskin, Mark Hancock, and Jesse Hoey. 2011. Extending interactions into hoverspace using reflected light. In Proceedings of the ACM International Conference on Interactive Tabletops and Surfaces - ITS '11, 262-263. https://doi.org/10.1145/2076354.2076406

[65] Yosra Rekik, Eric Vezzoli, Laurent Grisoni, and Frederic Giraud. 2017. Localized Haptic Texture: A Rendering Technique based on Taxels for High Density Tactile Feedback. In ACM Conference on Human Factors in Computing Systems (CHI '2017), 5006-5015.

[66] Tony Ro, Johanan Hsu, Nafi E. Yasar, L. Caitlin Elmore, and Michael S. Beauchamp. 2009. Sound enhances touch perception. Experimental Brain Research 195, 1: 135-143. https://doi.org/10.1007/s00221-0091759-8

[67] Deepak Ranjan Sahoo, Kasper Hornbaek, and Sriram Subramanian TableHop: An Actuated Fabric Display Using Transparent Electrodes. https://doi.org/10.1145/2858036.2858544

[68] Carsten Schwesig, Ivan Poupyrev, and Eijiro Mori. 2004. Gummi: a bendable computer. In Proceedings of the 2004 conference on Human factors in computing systems - CHI '04, 263-270. https://doi.org/10.1145/985692.985726

[69] Frederick Sebastian, Qiushi Fu, Marco Santello, and Panagiotis Polygerinos. 2017. Soft Robotic Haptic Interface with Variable Stiffness for Rehabilitation of Neurologically Impaired Hand Function. Frontiers in Robotics and AI 4: 69. https://doi.org/10.3389/frobt.2017.00069

[70] Rajinder Sodhi, Matthew Glisson, and Ivan Poupyrev. 2013. AIREAL: tactile gaming experiences in free air. In ACM SIGGRAPH 2013 Emerging Technologies on - SIGGRAPH '13, 1-1. https://doi.org/10.1145/2503368.2503370

[71] Jürgen Steimle, Andreas Jordt, and Pattie Maes. 2013. Flexpad: a highly flexible handheld display. In Proceedings of the SIGCHI Conference on Human Factors in Computing Systems - CHI '13, 237. https://doi.org/10.1145/2470654.2470688

[72] Paul Strohmeier, Jesse Burstyn, Juan Pablo Carrascal, Vincent Levesque, and Roel Vertegaal. 2016. ReFlex: A Flexible Smartphone with Active Haptic Feedback for Bend Input. In Proceedings of the TEI '16: Tenth International Conference on Tangible, Embedded, and Embodied Interaction - TEI '16, 185-192. https://doi.org/10.1145/2839462.2839494

[73] Paul Strohmeier and Kasper Hornbæk. 2017. Generating Haptic Textures with a Vibrotactile Actuator. In Proceedings of the $2017 \mathrm{CHI}$ Conference on Human Factors in Computing Systems - CHI '17, 4994-5005. https://doi.org/10.1145/3025453.3025812

[74] Sriram Subramanian, Dzimitry Aliakseyeu, and Andrés Lucero. 2006 Multi-layer interaction for digital tables. In Proceedings of the 19th annual ACM symposium on User interface software and technology - UIST '06, 269. https://doi.org/10.1145/1166253.1166295

[75] Suziah Sulaiman, Ann Blandford, and Paul Cairns. 2009. Haptic experience and the design of drawing interfaces. Interacting with Computers 22, 3: 193-2005.

[76] Chie Takahashi and Simon J Watt. 2017. Optimal visual-haptic integration with articulated tools. Experimental brain research 235, 5: 1361-1373. https://doi.org/10.1007/s00221-017-4896-5

[77] Ville Tikka and Pauli Laitinen. 2006. Designing Haptic Feedback for Touch Display: Experimental Study of Perceived Intensity and Integration of Haptic and Audio. Lecture Notes in Computer Science 4129: 36-44. https://doi.org/10.1007/11821731

[78] Hsin-Ruey Tsai and Jun Rekimoto. 2018. ElasticVR: Providing Multilevel Active and Passive Force Feedback in Virtual Reality Using Elasticity. In Extended Abstracts of the $2018 \mathrm{CHI}$ Conference on Human Factors in Computing Systems - CHI '18, 1-4. https://doi.org/10.1145/3170427.3186540

[79] Yon Visell, Keerthi Adithya Duraikkannan, and Vincent Hayward. 2014. A Device and Method for Multimodal Haptic Rendering of Volumetric Stiffness. Springer Berlin Heidelberg, 478-486. https://doi.org/10.1007/978-3-662-44193-0_60

[80] Quinglong Wang, Xiangshi Ren, Sayan Sarcar, and Xiaoying Sun. 2016. EV-Pen: Leveraging Electrovibration Haptic Feedback in Pen Interaction. In ACM International Conference on Interactive Surfaces and Spaces, 57-66.

[81] 81. Malte Weiss, Chat Wacharamanotham, Simon Voelker, and Jan Borchers. 2011. FingerFlux: near-surface haptic feedback on tabletops. In Proceedings of the 24th annual ACM symposium on User interface software and technology - UIST '11, 615. https://doi.org/10.1145/2047196.2047277 